\title{
Partial Hepatectomy
}

National Cancer Institute

\section{Source}

National Cancer Institute. Partial Hepatectomy. NCI Thesaurus. Code C15394.

Partial removal of the liver 